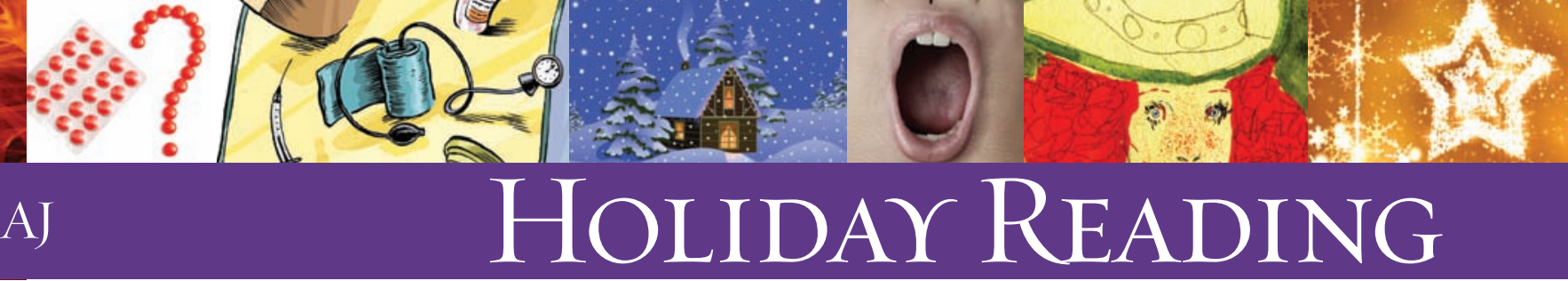

SATIRE

\title{
Cigarette smoking: an underused tool in high-performance endurance training
}

\author{
Kenneth A. Myers BSc
}

\begin{abstract}
The review paper is a staple of medical literature and, when well executed by an expert in the field, can provide a summary of literature that generates useful recommendations and new conceptualizations of a topic. However, if research results are selectively chosen, a review has the potential to create a convincing argument for a faulty hypothesis. Improper correlation or extrapolation of data can result in dangerously flawed conclusions. The following paper seeks to illustrate this point, using existing research to argue the hypothesis that cigarette smoking enhances endurance performance and should be incorporated into high-level training programs.
\end{abstract}

$\mathrm{P}$ erformances in endurance sports have been shrouded in controversy for the past 30 years because many athletes, intent on winning races and achieving faster times, have turned to banned performance-enhancing drugs. The World Anti-Doping Agency's 2009 list of prohibited substances and methods includes erythropoietin, other erythropoiesis-stimulating agents and various methods designed to enhance oxygen transfer. ${ }^{1}$ The effects of these agents and methods are thought to be primarily beneficial in endurance sports such as distance running and cycling. Despite the prohibition, the use of these drugs and techniques persists, as evidenced by continued positive results of drug tests in and out of competition.

Altitude training has also become common among endurance athletes, because it has been associated with an increase in performance and in serum hemoglobin and hematocrit levels. However, this response is transient - the physiologic variables return to their baseline soon after the athlete returns to sea level. ${ }^{2}$ This moderate performance benefit is outweighed by several severe and life-threatening risks, including pulmonary edema, ${ }^{3}$ cerebral edema ${ }^{4}$ and severe flatulence. ${ }^{5}$

While athletes endanger their careers and well-being in attempts to gain small benefits with illicit or inconvenient practices, a legal, nonprescription alternative has been largely ignored by athletes, coaches and exercise physiologists alike.

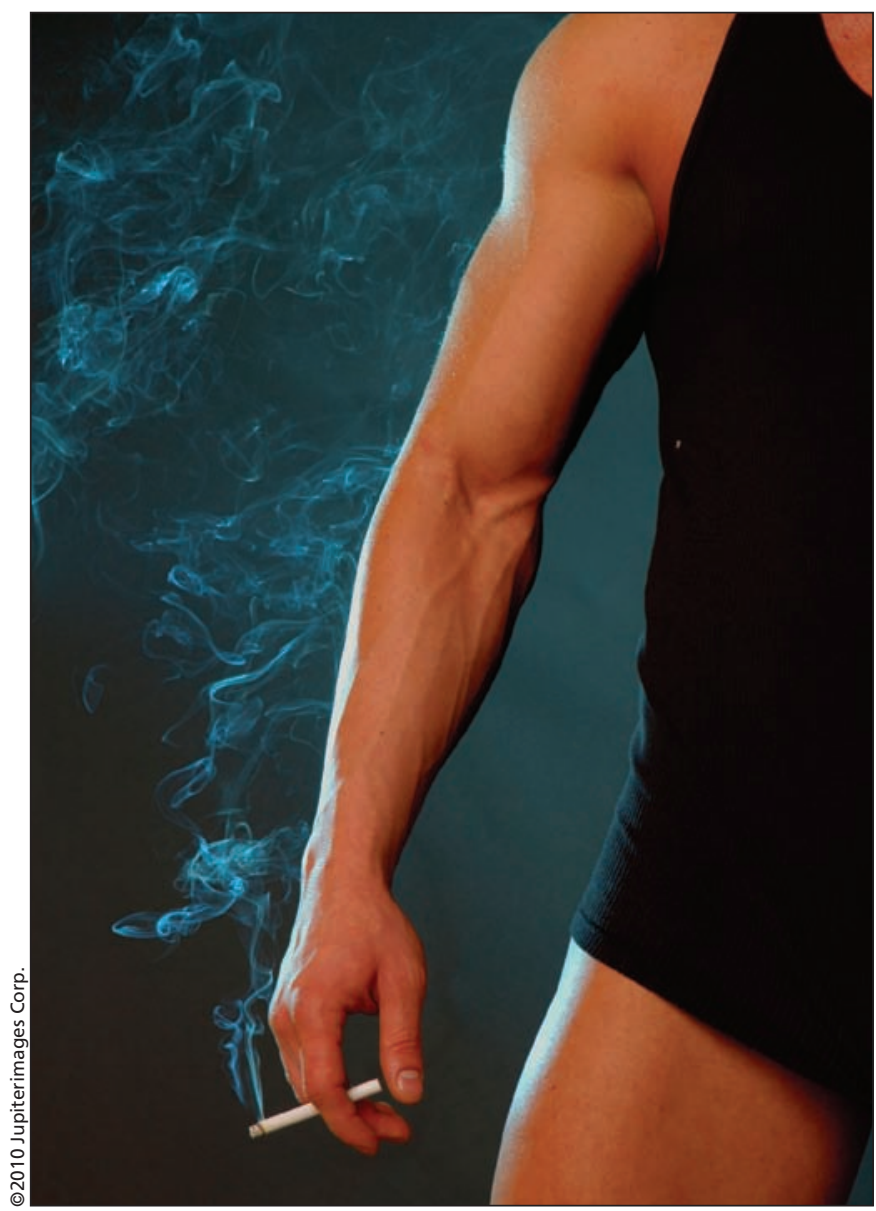

Cigarette smoking has been shown to increase serum hemoglobin and hematocrit levels, increase lung volume and stimulate weight loss - characteristics all known to enhance performance in endurance sports. This paper will discuss the potential benefits of cigarette smoking to endurance performance and make recommendations as to how individuals and national bodies could effectively integrate this practice into high-performance training programs.

From the Department of Medicine, University of Calgary, Calgary, Alta. 


\section{Factors related to endurance performance}

Cigarette smoking has an impact on three factors related to endurance performance: serum hemoglobin, lung volume and weight loss.

\section{Serum hemoglobin}

Blood hemoglobin concentration has been clearly associated with endurance performance and convincingly correlated with oxygen-carrying capacity. Decreased hemoglobin levels are associated with decreased maximal oxygen uptake and submaximal exercise capacity. ${ }^{6}$ Athletes have attempted to increase hemoglobin levels through blood doping, use of exogenous hormones and altitude training (real or simulated). ${ }^{7}$ These techniques are mostly illegal, and even altitude training has substantial health risks with physiologic benefits that are relatively modest and transient. Erythropoietin increases during the first 24-48 hours of altitude training, but levels normalize by one week. ${ }^{8}$ Although a three-week training stint at altitude results in a $7 \%-10 \%$ increase in hemoglobin, ${ }^{79}$ Heinicke and colleagues showed that levels return to normal within two weeks. ${ }^{9}$

In contrast, cigarette smoking has been associated with persistent increases in hemoglobin. Smoking 10 or more cigarettes per day is associated with an average hemoglobin increase of $3.5 \%$ compared with nonsmoking controls, a

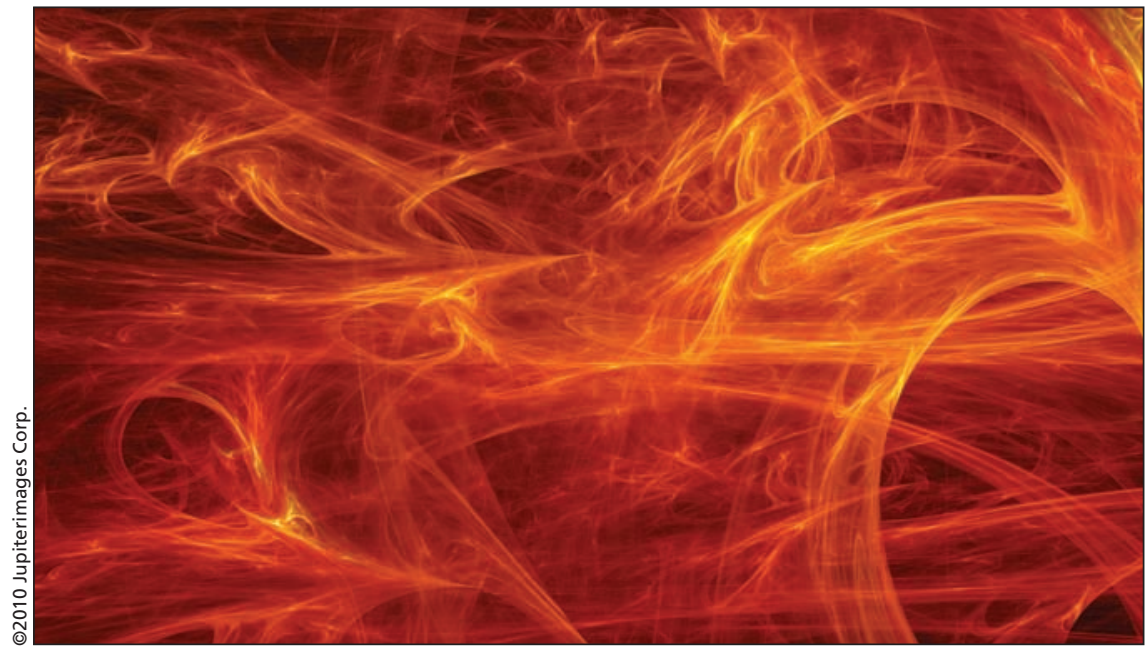

\section{Weight loss}

\section{Discussion}

smoking as part of a syndrome known as chronic obstructive pulmonary disease (COPD)..$^{14,15}$ These changes are not universal in smoking, however. Even with lifetime smoking, there is only a $50 \%$ chance of developing COPD.

Lower body weight is desirable in weight-bearing endurance sports, with distance running being the most obvious example. Cigarette smoking has been shown to stimulate weight loss through a variety of mechanisms including increased metabolic demand and appetite suppression. Hunger is suppressed via nicotine, the primary active ingredient in cigarettes. ${ }^{16}$ This could potentially be of great benefit to endurance athletes, because evidence suggests that exercise can increase appetite, putting athletes at greater risk for weight gain. ${ }^{17}$ Weight loss may be further enhanced in people who smoke cigarettes and go on to develop COPD. These people require more energy to breathe at rest and thus have increased resting energy requirements, often not balanced by caloric intake. ${ }^{18}$

Cigarette smoking has been shown to increase serum hemoglobin, increase total lung capacity and stimulate weight loss, factors that all contribute to enhanced performance in endurance sports. Despite this scientific evidence, the prevalence of smoking in elite athletes is actually many times lower than in the general population. ${ }^{19}$ The reasons for this are unclear; however, there has been little to no effort made on the part of national governing bodies to encourage smoking among athletes.

One important aspect of the benefits of smoking is they appear to be dose-dependent and may not develop until many years after initiation of treatment. With this in mind, smoking should be commenced at as young an age as is reasonably possible. Children who have not yet developed a pincer grasp might require modified cigarette holders, safety lighters or both. These points are moot at this time, because such initiatives are not possible in many countries because of existing legislation putting age restrictions on the change which can be maintained simply by continuing the treatment regimen. ${ }^{10}$ In fact, older people with longer smoking histories have higher hemoglobin levels, indicating a possible dose-dependent effect. ${ }^{10}$ The hemoglobin increases may be further enhanced with add-on therapy of ethanol, which also appears to act in a dose-dependent manner. ${ }^{10}$

\section{Lung volume}

Although lung volume measurement is not often used to determine endurance performance, increased total lung capacity has been shown to correlate with endurance training. Both swimming and long-distance running training result in increased total lung capacity. ${ }^{11-13}$ Results from other studies show that the same benefits can be derived from cigarette purchase of cigarettes. For example, the minimum age to buy cigarettes in Canada is 18 years. ${ }^{19}$

Although laws to restrict tobacco sales have existed in developed countries for some time, developing countries have only recently begun to pass similar legislation..$^{20}$ These latter countries have had much more success in endurance events in recent years, but research has not yet been conducted to investigate the prevalence of smoking among their top athletes and whether treatment was begun in childhood. More globally universal age restrictions may even the playing field with respect to endurance performance. However, countries should strongly consider repealing the existing laws or at least allowing exemptions for people thought to have athletic potential in endurance sports. 
In summary, existing literature supports the use of cigarettes to enhance endurance performance through weight loss and increased serum hemoglobin levels and lung volumes. However, athletes continue to neglect smoking and pursue illegal and dangerous methods that have only minor and transient effects on the same physiologic variables. More research is needed to clearly delineate when and how cigarette smoking should be integrated into high-performance training programs. Despite substantial federal financial support for endurance athlete development, we are aware of no such research programs at present.

\section{REFERENCES}

1. World Anti-Doping Agency. The prohibited list. Montréal (QC): The Agency 2009. p. 1-9.

2. Ingjer F, Myhre K. Physiological effects of altitude training on elite male crosscountry skiers. J Sports Sci 1992;10:37-47.

3. Stream JO, Grissom CK. Update on high-altitude pulmonary edema: pathogenesis, prevention, and treatment. Wilderness Environ Med 2008;19:293-303.

4. Wilson $\mathrm{MH}$, Newman S, Imray CH. The cerebral effects of ascent to high altitudes. Lancet Neurol 2009;8:175-91.

5. Auerbach P, Miller YE. High altitude flatus expulsion (HAFE). West J Med 1981; 134:173-4.

6. Calbet JA, Lundby C, Koskolou M, et al. Importance of hemoglobin concentration to exercise: acute manipulations. Respir Physiol Neurobiol 2006;151:132-40.

7. Saunders PU, Pyne DB, Gore CJ. Endurance training at altitude. High Alt Med Biol 2009;10:135-48.

8. Hahn AG, Gore CJ. The effect of altitude on cycling performance: a challenge to traditional concepts. Sports Med 2001;31:533-57.

9. Heinicke K, Heinicke I, Schmidt W, et al. A three-week traditional altitude training increases hemoglobin mass and red cell volume in elite biathlon athletes. Int $J$ Sports Med 2005;26:350-5.

10. Milman N, Pedersen AN. Blood haemoglobin concentrations are higher in smokers and heavy alcohol consumers than in non-smokers and abstainers: Should we adjust the reference range? Ann Hematol 2009;88:687-94.

11. Kaufmann DA, Swenson EW. Pulmonary changes during marathon training: a longitudinal study. Respiration 1981;41:217-23.

12. Courteix D, Obert P, Lecoq AM, et al. Effect of intensive swimming training on lung volumes, airway resistance and on the maximal expiratory flow-volume relationship in prepubertal girls. Eur J Appl Physiol Occup Physiol 1997;76:264-9.

13. Hagberg JM, Yerg JE II, Seals DR. Pulmonary function in young and older athletes and untrained men. J Appl Physiol 1988;65:101-5.

14. Colebatch HJ, Greaves IA, Ng CK. Pulmonary distensibility and ventilatory function in smokers. Bull Eur Physiopathol Respir 1985;21:439-47.

15. Petty TL, Silvers GW, Stanford RE. Mild emphysema is associated with reduced elastic recoil and increased lung size but not with air-flow limitation. Am Rev Respir Dis 1987;136:867-71.

16. Gross J, Stitzer ML. Nicotine replacement: ten-week effects on tobacco withdrawal symptoms. Psychopharmacology (Berl) 1989;98:334-41.

17. White LJ, Dressendorfer RH, Holland E, et al. Increased caloric intake soon after exercise in cold water. Int J Sport Nutr Exerc Metab 2005; 15:38-47.

18. Aniwidyaningsih W, Varraso R, Cano N, et al. Impact of nutritional status on body functioning in chronic obstructive pulmonary disease and how to intervene. Curr Opin Clin Nutr Metab Care 2008;11:435-42.

19. Alaranta A, Alaranta H, Patja K, et al. Snuff use and smoking in Finnish olympic athletes. Int J Sports Med 2006;27:581-6.

20. Itumbi D. Kenya enacts bold anti-smoking laws. Africa News. 2008 July 9. Available: www.africanews.com/site/Kenya_enacts_bold_antismoking_laws /list_messages/19353. (accessed 2009 Aug. 27).

Correspondence to: Kenneth Myers, Faculty of Medicine,

University of Calgary, 3330 Hospital Dr. NW Calgary AB

T2N 4N1; kmyers@ucalgary.ca 